\title{
PSYCHOPHYSIOLOGICAL FEATURES OF THE
}

FUNGTIONAL STATE TRANSFORMATION OF

COMBATANTS WITH CHRONIC FATIGUE DURING

THE REHABILITATION PERIOD, WITH RESPECT

TO THEIR PERSONAL OUALITIES

\author{
Kalnysh V. V., Zaitsev D. V.
}

\section{State Institution «Kundiiev Institute of Occupational Health of the National Academy of Medical Sciences of Ukraine», Kyiv}

Introduction. Personality traits can be correlated with burnout and occupational stress. Revealing such patterns can significantly improve the quality of professional selection and rehabilitation prognosis.

The aim of the study was to establish the influence of the personality profile on the success of the rehabilitation process of ATO/JFO veterans.

Materials and methods of research. 68 servicemen, men aged ( $32 \pm 5.6)$ years, participants of the Anti-Terrorist Operation Joint Forces Operation, who received comprehensive medical and psychological rehabilitation. Before and after rehabilitation, blood pressure and heart rate were measured. During rehabilitation, a survey was conducted on the tests of R. Cattell and SOPAS-8.

Results. Two subgroups of the surveyed were identified: subgroup 1 (46\%) and subgroup 2 (54\%). According to the Cattell test, the subgroups significantly differ in the scales «A», «G», «H», «Q1», «Q2», «Q3» (intellectual, emotional and communicative characteristics). According to the SOPAS-8 test, participants in subgroup 2 (compared with subgroup 1) have a lower level of desire for action, but a higher level of depression and exhaustion. Burnout symptoms in subgroup 2 individuals were more articulated. For subgroup 1, an unformed phase of voltage and the formation of a phase of resistance were typical, while for representatives of subgroup 2, mature phases of voltage and resistance. As a result of rehabilitation, the indicators of systolic and diastolic blood pressure significantly improve in all representatives. In subgroup 1, the level of connectivity of physiological parameters after rehabilitation decreases (the system of body functions regulation becomes more plastic). In subgroup 2, the connectivity of physiological parameters after rehabilitation drops sharply and loses its reliability (the plasticity of regulation increases sharply, and the degree of neuro-emotional stress falls more than in subgroup 1).

Conclusions. Personal qualities affect the restoration of the functional state of ATO/JFO veterans. An indicator of the connectivity of physiological functions, reflecting the activity of the cardiovascular system and emotional state may be used as indicator of the effectiveness of rehabilitation.

Key words: personal qualities, emotional state, emotional burnout, combatants, rehabilitation

\section{Introduction}

The problem of the influence of prolonged combat stress on the development of negative changes in the functional state of servicemen at different stages of their service is nowadays of great importance. According to some data, the formation of this condition can be influenced by the structure of personality. Thus, a number of studies on nurses, military personnel (infantry, aviation), firemen, policemen have shown that certain personality traits can correlate with emotional burnout and stress caused by professional activity conditions. In particular, extraversion reduces the risk of stress and posttraumatic disorder development [1,2], contributes to post-traumatic growth (accelerates recovery) [3]. Neuroticism, a personality trait characterized by emotional instability, anxiety, low self-esteem and autonomic disturbances, correlates positively with the phenomena of anxiety and depression $[2,4]$ and is a risk factor [5] or predictor [2,6-8] of workrelated stress. It has been repeatedly observed that consideration of such regularities makes it possible 
to improve considerably the accuracy of professional selection and to obtain an early rehabilitation prognosis in the presence of occupational diseases in servicepersons $[2,3,5,6]$. Nevertheless, the solution of the above issues is insufficiently and incompletely covered in the current literature, in particular when it comes to the rehabilitation of servicepersons.

Since over the years of the objective existence of constant combat encounters in the ATO/JFO zone the number of individuals who have developed negative shifts in their functional state has significantly increased, it is important to take into account the personality profile and the level of chronic fatigue development when conducting rehabilitation measures for servicemen, in particular combat veterans. Therefore, in order to further develop an adequate technology for veterans' health rehabilitation, it is necessary to establish indications of the influence of veterans' personality profile structure on the outcome and pace of rehabilitation.

The aim was to study the based on revealing the structure of personality traits and the degree of emotional burnout of ATO/JFO veterans, whose recovery of diseases and injuries takes place in the hospital conditions, to establish the influence of personality profile on the success of the rehabilitation process.

\section{Materials and methods of research}

Sixty-eight men aged ( $32 \pm 5.6)$, servicemen - participants of the Antiterrorist Operation - Joint Forces Operation (ATO/JFO) in East of Ukraine (duration of total participation in combat activities constituted $(320.5 \pm 25.5)$ days on average $)$ took part in the study. Between January 2019 and December 2020, the servicemen were receiving comprehensive medical and psychological rehabilitation for three weeks (including medication therapy, group psychotherapy, and physiotherapy) at the occupational disease clinic of the State Institution «Kundiiev Institute of Occupational Health of the National Academy of Medical Sciences of Ukraine». At the same time the combatants, who gave their informed voluntary consent, underwent psychophysiological monitoring. The sample was formed by the method of simple probability selection. The inclusion criteria were: direct participation in combat operations; a diagnosis of «posttraumatic stress disorder»(F43.1 in ICD-10) and/or «adaptation disorders» (F43.2 in ICD-10), (F43.2 according to ICD-10), as well as a criterion for exclusion of severe mental disorder (as defined in the Law of Ukraine «On Psychiatric Care»). For the study of individualpsychological, constitutional personality traits, multifactorial personality survey by R. Kettell [9] and SOPAS-8 survey [10] were used, which were compiled by the participants once. Physiological monitoring was performed by measuring blood pressure (BP) and heart rate (as well as derived parameters: pulse BP, Kérdö index) on the first and the last day of the rehabilitation course. Statistical analysis of the obtained data was performed by cluster analysis (k-means), correlation analysis; assessment of the reliability of the results was performed according to Student's t-test, Mann-Whitney U, $\chi^{2}$ test using STATISTICA 13.3 software package (StatSoft, USA; license AXA905I924220FAACD-N).

\section{Research results and their discussion}

Even the fastest revision of the values of personality assessments in the surveyed group indicates its heterogeneity. Therefore, for a detailed analysis of the personality profiles of the representatives of this group, a cluster analysis (k-means method) was used to divide the tested individuals into two subgroups. The result of the analysis indicated the presence of two subgroups in the group under study, almost equal in number: subgroup $1-46 \%$, and subgroup $2-54 \%$ of the examinees. 
The Mann-Whitney U-tests for the designated subgroups revealed a significant difference in the levels of certain scales: «A» $(p<0.005), \ll G »(p<$ $0.05), \ll \mathrm{H} \gg(\mathrm{p}<0.01), \ll \mathrm{Q} 1 »(\mathrm{p}<0.1), \ll \mathrm{Q} 2 »$ $(p<0.001), \ll Q 3 »(p<0.02)$. The values of the marks on these scales determine the intellectual peculiarities of the examined people ( $\ll \mathrm{Q} 1 »$ scale), their emotional-volitional properties ( $\mathrm{G} »$ scale, «Q3» scale), communicative properties and specific features of interpersonal interaction («A» scale, «H» scale, «Q2» scale). The established personality profiles of the participants in both subgroups are presented in Figure 1.

Subgroup 1 is characterized by the presence of particular manifestations of personality traits. The representatives of this subgroup are open, friendly, sociable and good-natured. They are characterized by a natural and easy-going demeanour, attentiveness, kindness and gentleness in relations. They willingly work with people, are active in eliminating conflicts, trusting, not afraid of criticism, experience vivid emotions, respond vividly to any events (the highest values on the «A» scale). Representatives of this subgroup are comparatively more patient, hardworking, emotionally mature and realistic. They are better able to obey the requirements of the group and are characterized by constancy of their interests. They have less pronounced nervous fatigue. In extreme cases, emotional rigidity and insensitivity (high G-scores) could be present. This subgroup is characterized by social courage, activity and readiness to deal with unfamiliar circumstances and people. They are risk-averse, free-spirited and disinhibited (highest on the «H» scale). High scores on the «Q $1 »$ scale characterize this subgroup as critical, with intellectual interests, analytical thinking and the desire to be well-informed. These people are more inclined to experiment, are more comfortable with new views and changes, do not trust authority, and do not take anything for granted. But the low value on the «Q2» scale defines their peculiarity: dependence on the group, following public opinion, striving to work and make decisions together with other people, orientation towards social approval. At the same time, these people often have little initiative in decision-making. At the same time, high scores on the «Q3» scale define developed self-control,

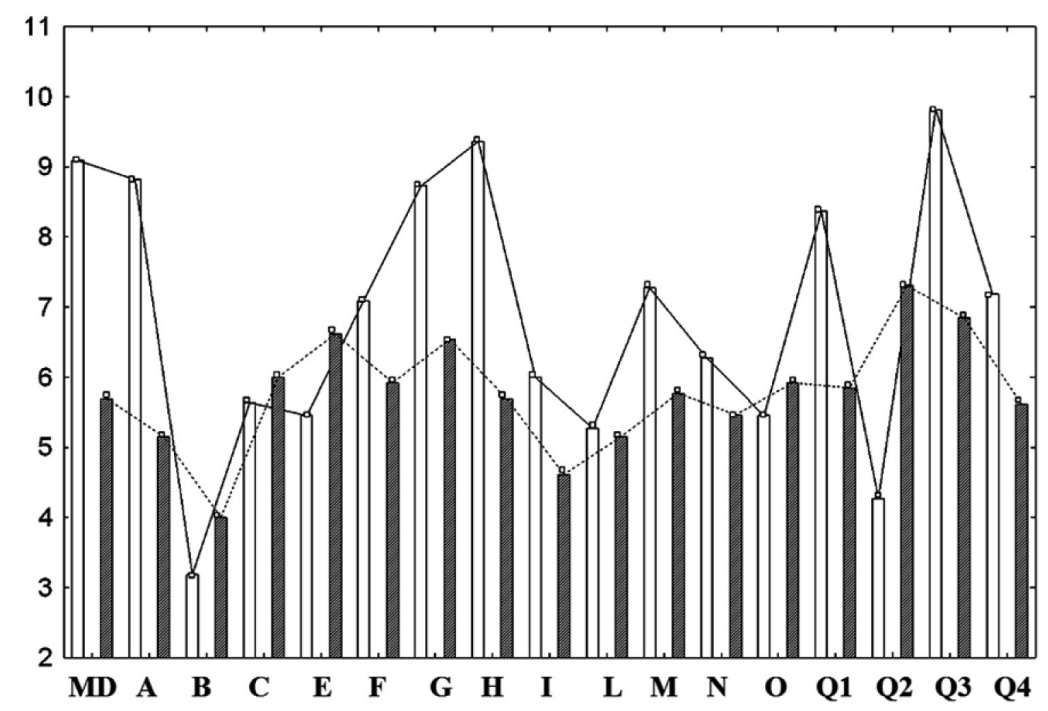

Figure 1. Personality profiles of combatants during their rehabilitation: unshaded bars represent the profile of combatants in subgroup 1; shaded bars represent the profile of combatants in subgroup 2; on the abscissa axis are the scales of the Cattell questionnaire; the ordinate axis is the mean values of the scales in scores 
accuracy in meeting social requirements. Such people follow personal self-image, control his or her emotions and behavior well and bring any matter to an end. A person of this subgroup is characterized by a goal-oriented and integrated personality.

The personality profile of those in subgroup 2 is very different from that of subgroup 1 . Individuals in subgroup 2 are unsociable, withdrawn, indifferent, have certain rigidity and are overly strict in their assessment of people. They are sceptical, cold to others, like solitude, and do not have close friends with whom they could be open (the lowest values on the «A» scale). In this case, there is an ultra-low tolerance for frustration, volatile interests, a tendency to mood lability, irritability, fatigue, neurotic symptoms, and hypochondria (lowest «G» scale values). Members of this subgroup are shy, insecure, reserved, timid and prefer to be in the shadows. Rather than having a large environment, they prefer to socialize with one or two persons, friends. They are characterized by an increased sensitivity to threat (the lowest values on the «H» scale). They are characterized by conservatism, resistance to traditional difficulties, they know what to believe in and do not seek new principles in the face of failure to manifest them, they are hesitant about new ideas and tend to moralize. They resist change and are not interested in analytical and intellectual considerations (the lowest values on the $\langle\mathrm{Q} 1 »$ scale). In this subgroup, people produce their own solutions, are independent, following their own chosen path. However, they are not always dominant: having their own opinion, they do not seek to impose it on others. This is not to say that they do not like people, but rather that they do not need the approval and support of others (highest values on the «Q2» scale). They are undisciplined, have an internal conflicted view of themselves and do not care about social demands (the lowest values on the «Q3» scale).

On the MD scale (self-esteem adequacy), the results for both subgroups did not differ significantly. However, the results for subgroup 1 were approximately 1.5 times higher, which may indicate that these individuals tend to overestimate their capabilities and overestimate themselves to a greater extent.

During the initial period of rehabilitation, the emotional state of servicepersons was also assessed. For

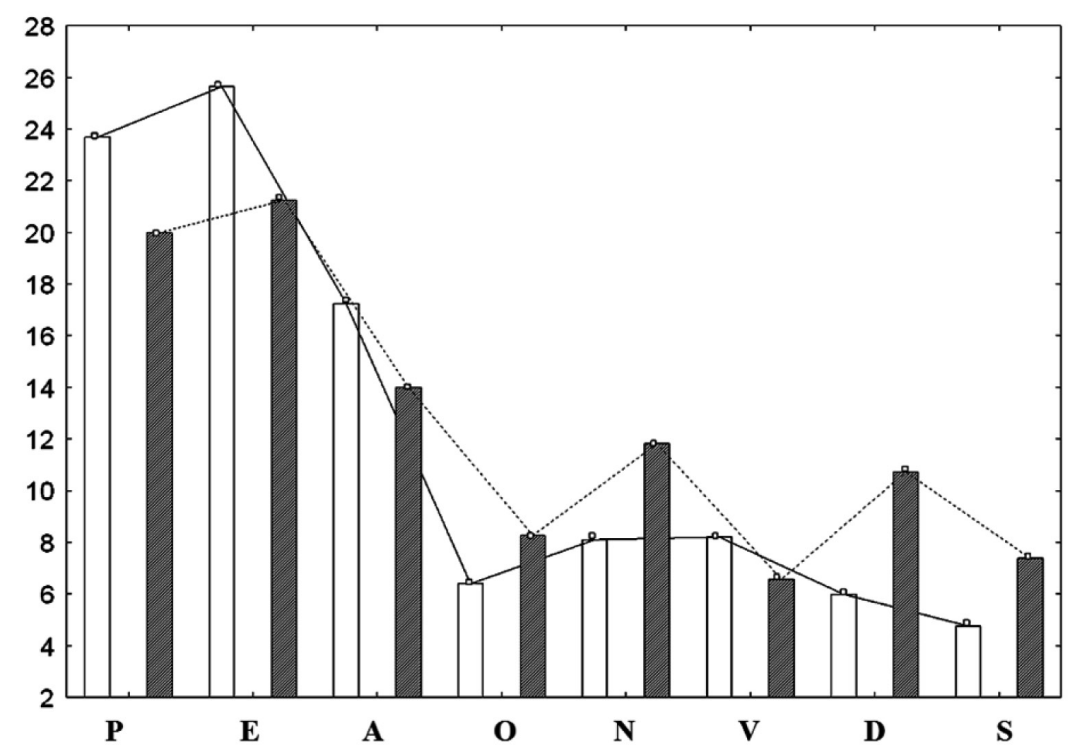

Figure 2. Profiles of combatants' emotional state during rehabilitation:

unshaded bars represent the profile of the combatants of subgroup 1; shaded bars represent the profile of the combatants of subgroup 2; the abscissa axis is the SOPAS-8 scale; the ordinate axis is the expression of emotional state as a percentage 
the purpose of this study, it is advisable to consider the emotional state of the two subgroups separately. The results of this analysis are shown in Figure 2.

The analysis of the received materials attracts attention to the fact that marks of positive emotions (scales $\mathrm{P}, \mathrm{E}$ and $\mathrm{A}$ ) in both subgroups have high values, but on other scales - low values that can testify to a favorable emotional background of the servicemen even at the beginning of rehabilitation. Radical differences (significant at the $\mathrm{p}<0.05$ Mann-Whitney U-criterion level for the A and D scales and $p<0.07$ for the $\mathrm{N}$ scale) were observed for three scales: A, N and D. Moreover, participants in subgroup 2 (as compared to subgroup 1) show lower levels of «strive for action» (A scale) - a positive emotion, but higher levels of «feelings of mental anxiety or resentment» ( $\mathrm{N}$ scale) and «mental depression and exhaustion» (D scale ) negative emotions. This proves that the emotional state of members of subgroup 1 is more favorable than that of members of subgroup 2 .
Since in the process of rehabilitation it is advisable to analyze the condition of the chronic fatigue of the servicemen, we considered the assessments of one of the aspects, indicating the level of the development of this condition - which is the emotional burnout. The results of estimating the components of emotional burnout of the participants in subgroups 1 and 2 are shown in Figure 3.

When comparing the severity of symptoms and phases of emotional burnout in subgroups 1 and 2 we can state that in subgroup 2 these symptoms are always more expressed. In the stress phase, the experience of psychotraumatic circumstances is «unformed» (8.2 points) in subgroup 1 and «mature» ( 19.1 points) in subgroup 2 , which characterizes the accumulation of despair and resentment. The symptom of dissatisfaction with oneself in subgroup 1 could be considered «unformed» (8.4 points) and «mature» in subgroup 2. The «cage-pulling» symptom for members of subgroups 1 and 2 could be interpreted as mature

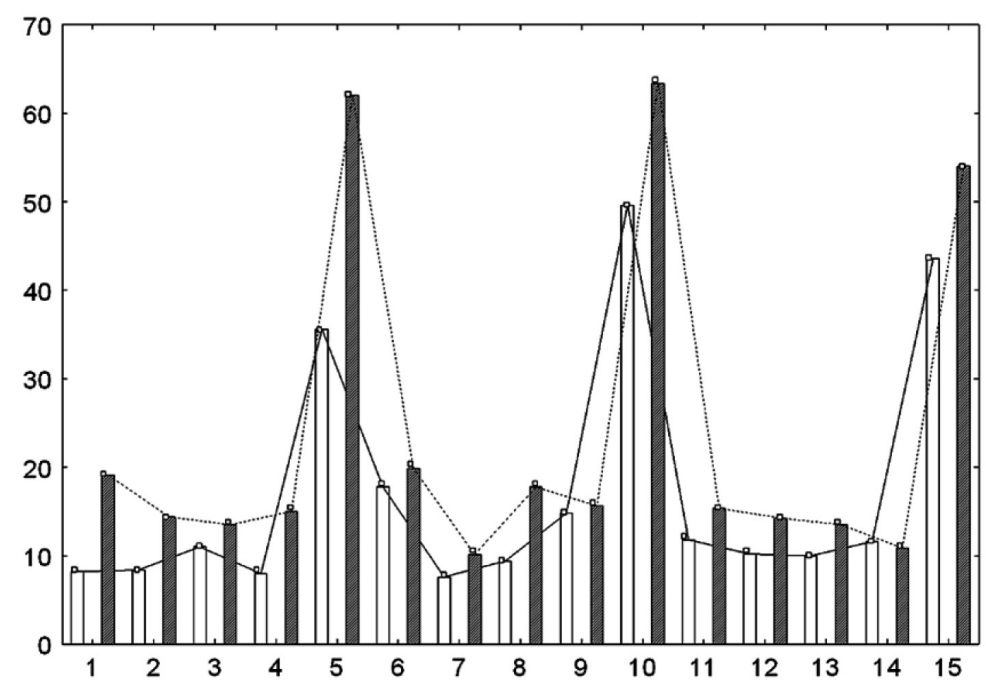

Figure 3. Profiles of combatants' emotional burnout during rehabilitation:

unshaded bars represent the profile of combatants of subgroup 1; shaded bars represent the profile of combatants of subgroup 2; on the abscissa axis there are the questionnaire scales of $V$. $V$. Boyko: 1 - experience of psychotraumatic circumstances, $2-$ dissatisfaction with oneself, 3 - cage-pulling, 4 - anxiety and depression, 5 - evaluation of «the tension» phase expression, 6 - inadequate selective emotional reaction, 7 - emotional and moral disorientation, 8-expansion of the sphere of energy saving, 9 - reduction of professional duties, 10 - evaluation of the «resistance» phase expression, 11 - emotional deficit, 12 - emotional detachment, 13 - personal detachment and depersonalization, 14 - psychoautonomic disorders, 15 - evaluation of the «exhaustion» phase expression; on an axis of ordinates - emotional burnout expression in points 
(11 and 13.5 pointsrespectively). The anxiety and depression symptom for subgroup 1 qualifies as «unformed» ( 8 points) and for subgroup 2 as «mature» (15 points). The cumulative level of the «tension» phase for subgroup 1 qualifies as «unformed» (35.6 points) and for subgroup 2 as «mature».

Estimates of the «resistance» phase acquire the following levels. In this case, the symptom of inadequate selective emotional response appears as «mature» in the form of emotional callousness, impoliteness and indifference towards other people for both subgroups (17.8 and 19.9 points respectively). The symptom of emotional and moral disorientation is «unformed» (7.6 points) in the representatives of subgroup 1 , and «mature» (10.1 points) in the individuals of subgroup 2. As for the symptom of expanding the sphere of saving emotions, it should be stated that for subgroup 1 it is «unformed» $(9$ points), whereas for subgroup 2 it is «mature» (17.8 points), and appears as fatigue from contacts, conversations, communication. Regarding the reduction in professional duties, the frequency of this symptom was similar in both subgroups and could be described as an «emerging symptom» (14.8 and 15.6 points respectively). Overall, there was a divergence of scores in the «resistance» phase. For subgroup 1 this phase is «in formation» (49.6 points), while for subgroup 2 the «resistance» phase is already «formed»(63.4 points). It clearly shows resistance to increasing stress.

All of the symptoms inherent in the «exhaustion» phase are rated as «forming» for both subgroups, and the final assessment of the level of development of this phase has a level corresponding to the «in formation» phase. Thus, the participants in the subgroups have different stages of phase formation. Subgroup 1 is characterized by an unformed «tension» phase, while the «resistance» phase is «in the process of formation». For subgroup 2, the «stress» and «resistance» phases are assessed as «mature».
For further analysis it is important to estimate reliability of difference between symptoms and levels of emotional burnout phases. By means of Mann-Whitney U-criterion it was established that there is a difference of estimations of experience of psychotraumatic circumstances at a level $p=0.027$ (the symptom is more expressed in subgroup 2), and the difference of levels of emotional displays in a phase «ension» has a tendency to increase in subgroup 2 ( $p=0.1)$. Thus, according to quantitative estimations, precursors of «triggering» mechanisms of emotional burnout formation are observed more in subgroup 2.

The domestic literature describes few studies in the field of personal characteristics and emotional state of servicepersons who served in the ATO/JFO. The study by M. M. Marusynets and E. V. Gubska that declares a number of interesting observations, in particular: «Stress resistance of ATO/JFO veterans is a key property that mediates the intensity of stressogenic factors (both acute and chronic) impact on personality and behavioral strategies. The development of a high level of stress tolerance is the basis of the psychophysical health of the military and an important factor ensuring their positive adaptation to civilian life. It has been found that veterans without severe wounds have a significantly higher level of stress resistance development, they are characterized by situational anxiety and an increased level of mediated aggression and irritability. Overall, virtually all average characteristics are within the normal range. Adaptive coping strategies of seeking social support are peculiar to the subjects, improving their process of resocialization in the new peaceful environment. The veterans who have experienced combat wounds demonstrate a low level of stress resistance and a significantly increased level of both personal and reactive anxiety. Aggressiveness, hostility and the prevalence of destructive coping-avoidance strategies are characteristic of the subjects. The identified personality 
characteristics are characteristic of individuals who may have a number of psychological problems and difficulties in adapting to civilian life» [11]. The difference in stress resilience of ATO/JFO veterans found here is associated with the severity of their injuries. However, the level of injury severity is a rather vague category. Therefore, no clear statistically proven shifts in stress tolerance and its components are indicated in this paper.

In our study, statistically significant differences in personality profiles are taken as the starting point for reflection. This technique allows us to make reasonable conclusions about the emotional state and emotional burnout of the ATO/JFO veterans undergoing rehabilitation.

Based on the results obtained, an analysis of the transformation of functional state of ATO/JFO veterans with regard to their personality profile was carried out. For the analysis it is reasonable to take the most popular and informative health indicators of the veterans who have suffered severe stress characteristics of the cardiovascular system activity: blood pressure, diastolic blood pressure, heart rate and their derivatives - pulse pressure and Kérdö index.

First of all, it is necessary to determine whether the rehabilitation procedures cause positive changes in the selected parameters during the whole period of rehabilitation. The results of such an analysis are given in Table 1 .
The table above shows that as a result of rehabilitation of ATO/JFO veterans diastolic blood pressure significantly improves and there is a weak tendency to improve systolic blood pressure and heart rate. Other parameters do not change significantly.

To solve the key posed question of the influence of personality traits on the quality of recovery of ATO/JFO veterans, the data of division of the studied group into two subgroups with different personality profiles, emotional state and the level of emotional burnout were involved. The results of the analysis of the established subgroups according to the indices of cardiovascular system functioning are presented in Table 2. It should be emphasized here that dividing into subgroups based on the available data on the personality profiles of each subgroup greatly reduced their number. Therefore, for further analysis, nonparametric criteria were applied, allowing us to disregard possible deviations of the data from the normal distribution.

The analysis showed that there was a significant improvement in systolic and diastolic BP and a marked tendency to reduce heart rate in both subgroups. This indicates a high effectiveness of the restorative measures but in most cases there is no specificity of the results of this improvement in any of the analyzed subgroups. The one exception is the change in the Kérdö index. First, there is a significantly higher level of influence of parasympathetic

Transformation of the levels of cardiovascular system functioning characteristics in ATO/JFO veterans during rehabilitation

\begin{tabular}{|l|c|c|c|}
\hline \multicolumn{1}{|c|}{ Indexes } & Before recovering & After recovery & $\boldsymbol{p}$ \\
\hline Systolic blood pressure, $\mathrm{mm} \mathrm{Hg}$ & $126.6 \pm 2.25$ & $122.2 \pm 1.60$ & $=0.11$ \\
\hline Diastolic blood pressure, $\mathrm{mm} \mathrm{Hg}$ & $81.7 \pm 1.32$ & $77.9 \pm 1.15$ & $=0.03$ \\
\hline Heart rate, 1/min & $73.0 \pm 1.18$ & $70.6 \pm 0.87$ & $=0.11$ \\
\hline Pulse pressure, mm Hg & $44.7 \pm 1.59$ & $44.2 \pm 1.15$ & $=1.17$ \\
\hline Kérdö index, unit & $-13.6 \pm 2.65$ & $-11.3 \pm 2.10$ & $=0.49$ \\
\hline
\end{tabular}

Note. $p$ - reliability of shifts in levels of indicators by Student's t-criterion. 
regulation of the autonomic nervous system in subgroup 1 at the initial stage of rehabilitation. Secondly, in the course of rehabilitation, the influence of parasympathetic regulation tends to decrease in the subgroup 1 and vice versa in the subgroup 2. All this testifies to the presence of unequal mechanisms of recovery of body functions of ATO/JFO veterans in subgroups with different personality profiles.

Further, a more in-depth analysis of the transformations of the functional state of ATO/JFO veterans in the process of rehabilitation was carried out. The fact is that the examined veterans, being in the zone of military operations, suffered a long and intensive stress. Their bodies developed significant emotional stress, especially, as shown earlier, in representatives of subgroup 2, where there was a greater degree of «mental anxiety» and «mental depression and exhaustion», as well as a significantly greater weakening of the regulatory action of the parasympathetic section of the autonomic nervous system.

As has been shown in other publications, increased neuro-emotional tension affects the tightness of correlational relationships between different psychophysiological characteristics of a person, referred to by the mathematical term «coherence» or «multicollinearity» [12]. The degree of coherence is assessed using a special coefficient of coherence (CC) based on the application of Criterion 2 to establish its reliability [13]. The coherence calculation of systolic BP, pulse pressure and heart rate gave the following results (Table 3 ).

When analyzing the data shown in Table 3 , it is noteworthy that in subgroup 1, the level of coherence of physiological parameters is significant in both cases. But after rehabilitation their CV decreased by $38 \%$ and the level of reliability of connectivity decreased from $\mathrm{p}<0.01$ to $\mathrm{p}<0.05$. Consequently, degrees of freedom of fluctuations of used physiological parameters after rehabilitation increase, the system of regulation of functions of an organism becomes more plastic. At the same time, a more striking picture is observed in subgroup 2 . While before the application of rehabilitation techniques, CP was reliably high (approximately equal to that in subgroup 1), after rehabilitation, it drops sharply by $79 \%$ and ceases to be reliable. In other

Changes in cardiovascular system characteristics in ATO/JFO veterans with different

Tahle 2 personality profiles before and after rehabilitation

\begin{tabular}{|c|c|c|c|c|}
\hline \multirow{2}{*}{ Indexes } & \multicolumn{2}{|c|}{ Subgroup 1, $M e$} & \multicolumn{2}{|c|}{ Subgroup 2, Me } \\
\hline & Before recovery & After recovery & Before recovery & After recovery \\
\hline $\begin{array}{l}\text { Systolic blood pressure, } \\
\mathrm{mm} \mathrm{Hg}\end{array}$ & $\begin{array}{c}135.5 \\
{[110-150]}\end{array}$ & $\begin{array}{c}127.5 \\
{[110-140]^{\wedge}}\end{array}$ & $\begin{array}{c}130.0 \\
{[110-150]}\end{array}$ & $\begin{array}{c}120.0 \\
{[110-130]^{\wedge}}\end{array}$ \\
\hline $\begin{array}{l}\text { Diastolic blood pressure, } \\
\mathrm{mm} \mathrm{Hg}\end{array}$ & $\begin{array}{c}80.0 \\
{[80-90]}\end{array}$ & $\begin{array}{c}79.0 \\
{[65-90]^{\wedge}}\end{array}$ & $\begin{array}{c}80.0 \\
{[80-100]}\end{array}$ & $\begin{array}{c}79.0 \\
{[70-80]^{\wedge}}\end{array}$ \\
\hline Heart rate, $1 / \mathrm{min}$ & $\begin{array}{c}75.0 \\
{[63-80]}\end{array}$ & $\begin{array}{c}72.0 \\
{[66-78]^{\circ}}\end{array}$ & $\begin{array}{c}74.0 \\
{[68-92]}\end{array}$ & $\begin{array}{c}68.0 \\
{[62-72]^{\circ}}\end{array}$ \\
\hline Pulse pressure, $\mathrm{mm} \mathrm{Hg}$ & $\begin{array}{c}55.5 \\
{[30-60]}\end{array}$ & $\begin{array}{c}50.0 \\
{[35-55]}\end{array}$ & $\begin{array}{c}40.0 \\
{[40-65]}\end{array}$ & $\begin{array}{c}40.0 \\
{[40-50]}\end{array}$ \\
\hline Kérdö Index, unit & $\begin{array}{c}-11.2 \\
{[-31.5-(-1.3)]}\end{array}$ & $\begin{array}{c}-7.4 \\
{[-26.8-5.9]}\end{array}$ & $\begin{array}{c}-5.3 \\
{[-47.1-13.0]^{*}}\end{array}$ & $\begin{array}{c}-12.9 \\
{[-29.0-0.0]}\end{array}$ \\
\hline
\end{tabular}

Note. ${ }^{\wedge},{ }^{\circ}$ Reliability of shifts in indicators before and after recovery for each of the subgroups by paired Wilcoxon test at $p<0.05$ and $p<0.1$, respectively, * reliability of shifts in indicators before recovery for subgroups 1 and 2 by Mann-Whitney $U$-criterion at $p<0.05$, Me - median, in square brackets are 10 and 90 percentiles respectively. 
ISSN 2223-6775, Ukrainian Journal of Occupational Health, 2021, 17 (4), 225-234

Table 3

Stress level of regulatory systems in the body of ATO/JFO veterans before and after rehabilitation

\begin{tabular}{|l|c|c|c|c|}
\hline \multirow{2}{*}{ The term of the study } & \multicolumn{2}{|c|}{ Subgroup 1 } & \multicolumn{2}{c|}{ Subgroup 2 } \\
\cline { 2 - 5 } & CC & $\boldsymbol{p}$ & CC & \multicolumn{1}{c|}{} \\
\hline Before recovery & 14.48 & $<0.01$ & 12.53 & $>0.01$ \\
\hline After recovery & 8.91 & $<0.05$ & 2.64 & 0.1 \\
\hline
\end{tabular}

Note. CC is coefficient of coherence; $p$ is the level of reliability of connectivity of cardiovascular parameters, calculated using the $\chi^{2}$ criterion.

words, the plasticity of the physiological regulatory processes in this subgroup sharply increases, and the degree of neuro-emotional tension in the body of the members of this subgroup decreases much more than in subgroup 1.

As it was established earlier, the members of subgroup 1 are characterized by openness, communicativeness, they are not afraid of criticism, experience vivid emotions, and respond vividly to any events. The representatives of this subgroup are characterized by social courage, activity, and readiness to deal with unfamiliar circumstances and people. It can be assumed that such personality traits cause higher neuro-emotional tension in a person, which manifests itself at the physiological level. In the process of restoration of the functional state this tension at them, certainly, somewhat decreases. However, an active way of life does not allow them to «extinguish» the tension to a large extent, since courageous, considerably emotionally colored actions in their further life activities await them.

In addition, subgroup 2 is characterized by traits of indifference, rigidity, conservatism, resistance to traditional difficulties, knowing what to believe in, resistance to change and a lack of interest in analytical and intellectual considerations. On the physiological level, despite outward sensitivity, a greater plasticity of adaptive reactions is formed, which contributes to a rapid and sustainable recovery of their functional state, despite the «worst» emotional state and a greater level of emotional burnout at the beginning of rehabilitation.
Thus, the analysis suggests that personal qualities have an impact on the recovery process of the functional state of ATO/JFO veterans. This influence is not particularly pronounced and is almost imperceptible when viewed superficially. However, at the initial stage of recovery, a significantly lower level of the Kérdö index in the representatives of subgroup 2 is noticeable, which suggests a greater predominance of the regulatory function of the parasympathetic nervous system in them. Closer examination of mechanisms of regulation of physiological functions by establishing their coherence reveals a significant difference in recovery outcomes for individuals with more conservative, stable views of events. From a practical perspective, knowledge of a veteran's psychological status will enable the clinician to approach the rehabilitation process in a more meaningful way, considering the role of personality traits in influencing the pace and effectiveness of recovery measures.

This study opens up new perspectives and possibilities for analyzing the influence of personality traits on a person's functional status, particularly in the rehabilitation process. Only some of the more accessible indicators of a person's functional state have been used in this study. It will be useful in the future to considerably extend the list of such physiological and psychophysiological indicators in order to clarify the subtle mechanisms of recovery of organism functions after severe stress and develop rational rehabilitation technologies taking into account the personality traits of the person. 


\section{Conclusions}

1. It has been established, that the personal qualities of a person influence the level of functional recovery of ATO/JFO veterans. To a greater extent this positive influence is inherent in persons with traits of indifference, rigidity, conservatism of views, resistance to traditional difficulties, who can resist changes and are not interested in analytical and intellectual conside-

\section{References}

1. Chung, I. S., Lee, M. Y., Jung, S. W. and Nam, C. W. (2015), "Minnesota multiphasic personality inventory as related factor for post traumatic stress disorder symptoms according to job stress level in experienced firefighters: 5-year study", Ann Occup Environ Med, No. 27, p. 16, https://doi.org/10.1186/s40557-015-0067-y.

2. Wang, Y. and Zhang, B. (2017), "Impact of personality trait and professional identity on work-related depression, anxiety and irritation among chinese nurses", Southeast Asian J Trop Med Public Health, Vol. 48, No. 2, pp. 447-454.

3. Yang, S. K. and Ha, Y. (2019), "Predicting posttraumatic growth among firefighters: the role of deliberate rumination and problem-focused coping", Int $J$ Environ Res Public Health, Vol. 16, No. 20, p. 3879, https://doi.org/10.3390/ijerph16203879.

4. Hansell, N. K., Wright, M. J., Medland, S. E., Davenport, T. A., Wray, N. R., Martin, N. G. and Hickie, I. B. (2012), "Genetic co-morbidity between neuroticism, anxiety/depression and somatic distress in a population sample of adolescent and young adult twins", Psychol Med, Vol. 42, No. 6, pp. 1249-1260, https://doi.org/ 10.1017/S0033291711002431.

5. Tyssen, R., Vaglum, P., Grønvold, N. T. and Ekeberg, Ø. (2005), "The relative importance of individual and organizational factors for the prevention of job stress during internship: a nationwide and prospective study", Med Teach, Vol. 27, No. 8, pp. 726-731, https://doi. org/10.1080/01421590500314561.

6. Yusof, Z. Y. M., Hassan, W. N. W., Razak, I. A., Hashim, S. M. N., Tahir, M. K. A. M. and Keng, S. B. (2016), rations. Even in the initial phase of rehabilitation, they have a more pronounced influence of the parasympathetic part of the autonomic nervous system.

2. It is shown that a reliable indicator of rehabilitation efficiency can serve as an indicator of coherence of physiological functions reflecting activity of cardiovascular system (systolic and pulse blood pressure, heart rate) and reflecting emotional condition of a person.

"Personality traits and stress levels among senior dental students: evidence from Malaysia and Singapore", Southeast Asian JTrop Med Public Health, Vol. 47, No. 6, pp. 1353-1365.

7. Cabarkapa, M., Korica, V. and Rodjenkov, S. (2011), "[Personal traits and a sense of job-related stress in a military aviation crew]", [Article in Serbian], Vojnosanit Pregl., Vol. 68, No. 2, pp. 143-149, https://doi.org/ 10.2298/VSP1102143C.

8. Leblanc, J., Ducharme, M. B. and Thompson, M. (2004), "Study on the correlation of the autonomic nervous system responses to a stressor of high discomfort with personality traits", Physiol Behav, Vol. 82, No. 4, pp. 647652, https://doi.org/10.1016/j.physbeh.2004.05.014.

9. Rajgorodskij, D. Ya. (ed.) (2000), Prakticheskaya psikhodiagnostika. Metodiki i testy`. Uchebnoe posobie [Practical psychodiagnostics. Techniques and tests. Tutorial], BAKhHRAKH-M, Samara, Russia.

10. Sopov, V. F. (2005), Psikhicheskie sostoyaniya v napryazhyonnoj professional'noj deyatel'nosti [Mental states in intense professional activity], Akademicheskij Proekt, Moscow, Russia.

11. Marusy`necz`, M. M. and Gubs`ka, Ye. V. (2020), "Stress resilience of ATO/JFO veterans: an empirical context of the study", Current issues of psychology, Vol. 9, No. 13, pp. 210-220.

12. Kal'nish, V. V. (1994), "Changes in the voltage of the body subsystems in personsworking in shifts», Occupational medicine and industrial ecology, No. 11, pp. 36-39.

13. Fyorster, E' and Ryoncz, Yu. (1983), Metody korrelyaczionnogo i regressionnogo analiza [Correlation and regression analysis methods], Finansy`i statistika, Moscow, USSR.

\section{ORCID ID of co-authors and their contribution to the preparation and writing of the article:}

Kalnysh V. V. (ORCID ID 0000-0002-5033-6659) - hypothesis, mathematical analysis, conclusions;

Zaitsev D. V. (ORCID ID 0000000208411504) - search and analysis of literature, collection of primary data, text.

Information on the source of funding for the study: research is part of research work «Install the patterns of transformation of chronic fatigue in the rehabilitation period in combatants after the action of combat stress», No. of state registration $0119 \mathrm{U} 100294$.

Received: November 10, 2021

Accepted for publication: December 9, 2021

Contact person: Kalnysh Valentyn, senior researcher, laboratory of psychophysiology of labor, SI «Kundiiev Institute of Occupational Health of the National Academy of Medical Sciences of Ukraine», 75, Saksagansky str., Kyiv, 01033. Tel.: + 3806734881 43. E-mail: vkalnysh@ukr.net 